\title{
Waveguide for cold atoms: Spin-1 magnetic particles and a filamentary current
}

\section{Citation}

Berg-Sorensen, Kirstine, Michael M. Burns, J. A. Golovchenko, and Lene Vestergaard Hau. 1996. Waveguide for Cold Atoms: Spin-1 Magnetic Particles and a Filamentary Current. Physical Review A 53, no. 3: 1653-1667. doi:10.1103/physreva.53.1653.

\section{Published Version}

doi:10.1103/PhysRevA.53.1653

\section{Permanent link}

http://nrs.harvard.edu/urn-3:HUL.InstRepos:29406261

\section{Terms of Use}

This article was downloaded from Harvard University's DASH repository, and is made available under the terms and conditions applicable to Other Posted Material, as set forth at http:// nrs.harvard.edu/urn-3:HUL.InstRepos:dash.current.terms-of-use\#LAA

\section{Share Your Story}

The Harvard community has made this article openly available.

Please share how this access benefits you. Submit a story.

\section{Accessibility}




\title{
Waveguide for cold atoms: Spin-1 magnetic particles and a filamentary current
}

\author{
Kirstine Berg-Sørensen, ${ }^{1}$ Michael M. Burns, ${ }^{1}$ J. A. Golovchenko, ${ }^{1,2}$ and Lene Vestergaard Hau ${ }^{1,2}$ \\ ${ }^{1}$ The Rowland Institute for Science, 100 Edwin H. Land Boulevard, Cambridge, Massachusetts 02142 \\ ${ }^{2}$ Lyman Laboratory, Harvard University, Cambridge, Massachusetts 02138
}

(Received 31 August 1995)

\begin{abstract}
We consider a waveguide for cold neutral atoms with a magnetic moment proportional to their spin angular momentum. The waveguide consists of a thin wire carrying a constant current. For the spin- 1 case, we find bound states for the two-dimensional part of the motion around the wire, and present numerical and approximate analytic results for these bound states. In an experiment, the bound states can decay due to various effects, and we calculate the time scales involved.
\end{abstract}

PACS number(s): 03.75.Be, 03.65.Ge, 32.80.Pj, 52.55.Lf

\section{INTRODUCTION}

Within the past decade atoms cooled to very low temperatures by laser cooling have become widely available $[1,2]$, so that new areas of research have become accessible. Cold atoms in optical molasses can be obtained with de Broglie wavelengths on the order of the wavelength of visible light $[3,4]$. A very direct way of studying the wave nature of these cold atoms is by capturing them in waveguides. Two such waveguides for neutral atoms have been proposed [5-7], based on the interaction of cold atoms with either a charged wire or a current carrying wire.

In this paper we discuss one of these waveguides, which consists of a thin wire carrying a constant current $I$. An atom of magnetic moment $\boldsymbol{\mu} \propto \mathbf{S}$ couples to the magnetic field from the thin wire, and its motion perpendicular to the wire can be bound. This waveguide was first suggested for neutrons [8], and recently for atoms treated as spin- $1 / 2$ particles [5]. In Ref. [5], energy eigenvalues and eigenfunctions were found analytically by a supersymmetric factorization [9-12]. A good quantum number $m$ for the atoms around the wire is connected with the projection of the total angular momentum along the wire, $m \hbar$. Only for $m=0$ in the spin- 1 case can an algebraic analytic energy spectrum be obtained using arguments analogous to the spin- $1 / 2$ case. Such solutions are readily available from the results in [5]. We shall discuss our attempts to extend the supersymmetry technique used for $s=1 / 2$ to the entire spin- 1 case.

We present analytic approximations and numerical solutions of the spin-1 system. The study of a spin-1 system is motivated by the availability of cold bosonic alkali-metal atoms like sodium, which has an $F=1$ ground state.

This paper is organized as follows: in Sec. II, we define the problem and separate the Hamiltonian into contributions from the various degrees of freedom of the system; we also briefly discuss the supersymmetry technique. Then, in Sec. III, we present the physical picture of atom binding in the classical limit, and in Sec. IV we give our numerical results. It turns out that the analytic solution from supersymmetric quantum mechanics applied to the spin-1/2 system [5] is applicable only approximately in the spin- 1 case. Furthermore, the numerical energy eigenvalues are not degenerate in the angular quantum number $m$ as in the spin-1/2 system, indicating breaking of the supersymmetry in going from $s=1 / 2$ to $s=1$. Nevertheless, the numerical energy eigenvalues are almost degenerate, and we have used this as a guide to search for approximate analytic solutions, which are presented in Sec. V. In Sec. VI, we compare these approximate analytic results with the numerical ones. Finally, in Sec. VII, we study the mechanisms that will lead to decay of the trapped states and calculate the corresponding life times.

\section{HAMILTONIAN}

We study a particle of mass $M$ with magnetic moment $\boldsymbol{\mu}$ moving in the magnetic field of a current carrying wire. The bound states are found as the negative energy eigenstates of the stationary Schrödinger equation

$$
-\frac{\hbar^{2}}{2 M} \nabla^{2} \psi-\boldsymbol{\mu} \cdot \mathbf{B} \psi=E \psi
$$

The magnetic moment of the particle is given by

$$
\boldsymbol{\mu}=-g \mu_{B} \mathbf{S} / \hbar,
$$

where $g$ is the Lande $g$ factor and $\mu_{B}$ is the Bohr magneton. Let the wire define the $x$ axis, with the current $I$ flowing in the positive $x$ direction; then the magnetic field from the wire, in cylindrical coordinates $(x, r, \phi)$, is

$$
\mathbf{B}(r, \phi)=\frac{2 I}{c r}\left(\mathbf{e}_{z} \cos \phi-\mathbf{e}_{y} \sin \phi\right),
$$

where $\mathbf{e}_{i}, i=x, y, z$, are the unit vectors of the Cartesian coordinate system.

In the adiabatic limit, where the Larmor precession frequency $\omega_{L}$ is much larger than the orbital frequency around the wire, $\omega_{\text {orbit }}$, the projection of the particle's magnetic dipole on the direction of the magnetic field is constant, and the particle is bound in a scalar $1 / r$ potential. As explained previously [5], it is then convenient to use the local direction of the magnetic field as the spin quantization axis, accomplished by applying the unitary operator $\exp \left(i S_{x} \phi / \hbar\right)$. After this transformation, the Schrödinger equation (1) separates in $\phi$ and $r$, so we can write the total wave function in the new representation as 


$$
\Psi(x, r, \phi)=e^{i m \phi} e^{i k x} \frac{\chi(r)}{\sqrt{r}} .
$$

The angular part, $e^{i m \phi}$, is an eigenfunction for the total angular momentum along the direction of the wire, represented by the operator $-i \hbar(\partial / \partial \phi)$, with eigenvalue $\hbar m$, where $m$ is an integer for a particle of integer $s$ and half-integer for a particle of half-integer $s$. The radial equation to solve for the spinor function $\chi(r), H_{m} \chi=\epsilon \chi$, is (in dimensionless units)

$$
-\frac{d^{2}}{d \rho^{2}} \chi+\frac{1}{\rho^{2}}\left\{\left(\frac{S_{x}}{\hbar}-m\right)^{2}-\frac{1}{4}\right\} \chi+\frac{2}{\rho} \frac{S_{z}}{\hbar} \chi=\epsilon \chi .
$$

Here, the reduced coordinate is $\rho=r / r_{0}$, and the reduced transverse energy is $\epsilon$, where

$$
r_{0}=\frac{\hbar^{2}}{M C^{2}}, \quad C^{2}=\frac{2 g \mu_{B} I}{c}, \quad \epsilon=\frac{E-(\hbar k)^{2} /(2 M)}{C^{2} /\left(2 r_{0}\right)} .
$$

The wave function $\chi$ must be normalizable and must vanish at $\rho=0$. The potential has a singularity at $\rho=0$ and, to ensure that the kinetic energy operator is Hermitian, we must further require that $\chi(\rho) / \sqrt{\rho} \rightarrow 0$ as $\rho \rightarrow 0$. (See [13] and Appendix A.)

So far the description is independent of the actual spin value for the particle, and is identical to the outline in [5]. As described in that paper, for a spin-1/2 particle, the radial equation (5) can be solved analytically by supersymmetric quantum mechanics. This special symmetry of the spin-1/2 problem also shows up through the existence of a dynamic symmetry group and an extra constant of the motion similar to the Runge-Lenz vector in the scalar hydrogen atom [14]. For the spin-1 system the factorization used in supersymmetric quantum mechanics is no longer exact: although for values of $m \neq 0$ we can "factorize" the radial Hamiltonian $H_{m}$,

$$
H_{m}=A_{m}^{\dagger} A_{m}+K_{m},
$$

with

$$
A_{m}=\frac{d}{d \rho}+W_{m}(\rho) ; \quad A_{m}^{\dagger}=-\frac{d}{d \rho}+W_{m}(\rho),
$$

and arrange for the supersymmetric partner Hamiltonian,

$$
H_{m}^{(+)}=A_{m} A_{m}^{\dagger}+K_{m},
$$

to equal $H_{m+1}$, the constant matrix $K_{m}$ is not diagonal, and so we cannot interpret the solution of $A_{m} \chi=0$ as the ground state wave function. Note for later reference that the matrix $K_{m}$ has a twofold degenerate eigenvalue,

$$
\varepsilon_{1}=-\frac{1}{2 m^{2}}-\frac{1}{2(m+1)^{2}}
$$

and a second eigenvalue $\varepsilon_{2}=0$. Also note that the direct connection between Hamiltonians of different $m$ 's through $H_{m}^{(+)}=H_{m+1}$ is not necessary for supersymmetric quantum mechanics to be applicable, but that special property was found in the spin-1/2 system and it implies a degeneracy in the energy eigenvalues for the $n$th excited state of $H_{m}$ and the $(n-1)$ th excited state of $H_{m+1}$.

As mentioned earlier, in the special case of $m=0$, the equations for the $\chi^{( \pm)}$components of the spinor wave functions (superscripts, \pm 0 correspond to eigenvalues $\pm 1,0$ of $\left.S_{z} / \hbar\right)$ reduce, after a simple scaling of the coordinate, to the equations for the two spinor components in the spin-1/2 case, with $m_{1 / 2}=-1 / 2$ [5]. The $\chi^{(0)}$ component is not coupled to the two $\chi^{( \pm)}$components and it may be chosen to vanish. Due to the direct mapping between the coupled system of equations for the $\chi^{( \pm)}$components in the $m=0$, spin-1 case, to the spin-1/2 system, we know the eigenfunctions and energy eigenvalues of this system analytically, from Ref. [5]. For example, the energy eigenvalue for the $N$ th excited state of $m=0$ is

$$
\epsilon_{N, m=0}=-\frac{1}{(1+N)^{2}} \text {. }
$$

Below, numerical and approximate analytic results for all other values of $m$ will be presented. Since the system is invariant with respect to a change of sign of $m$, we consider only positive values of $m$.

\section{ADIABATIC LIMIT}

In the adiabatic limit, where the precession frequency of the magnetic moment around the field is large compared to the orbital frequency of the atom around the wire, $\omega_{L} \gg \omega_{\text {orbit }}$, the projection of $\boldsymbol{\mu}$ on the magnetic field vector $\mathbf{B}$ is approximately constant. In this case, one spinor component in the rotating spin basis is bound in a scalar $1 / r$ potential (cf. also [5,7]), and it corresponds to the experiments in the classical regime reported in [15]. The adiabatic Hamiltonian is found by neglecting the kinetic energy associated with rotation of the spin direction, i.e., in the transformed basis, by neglecting the terms with $S_{x}$ and $S_{x}^{2}$ in Eq. (5). We are then left with

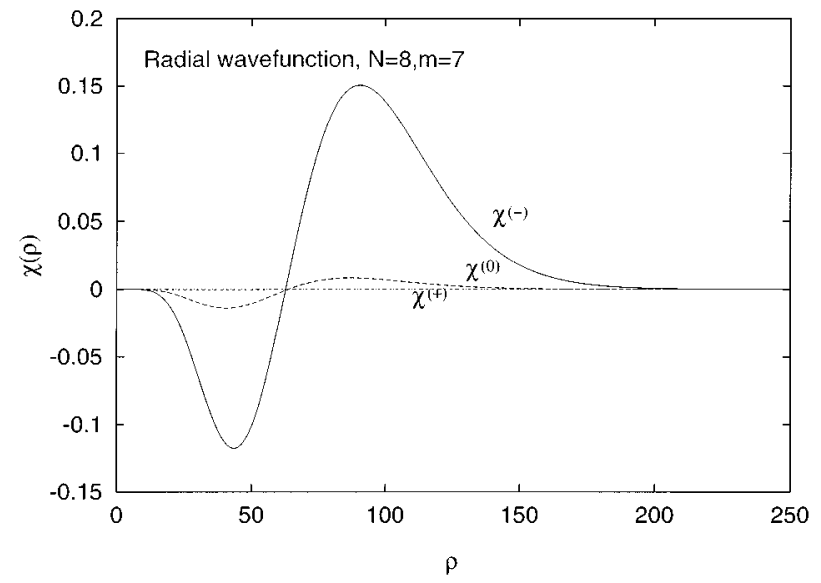

FIG. 1. The numerically calculated eigenfunction $\chi(\rho)$ for $N=8, m=7$. The largest component, $\chi^{(-)}$, has the eigenvalue $-\hbar$ for the spin operator $S_{z}$, the next component, $\chi^{(0)}$, has eigenvalue 0 , and the smallest component, $\chi^{(+)}$, has eigenvalue $\hbar$. The $\chi^{(+)}$component is hardly visible. 


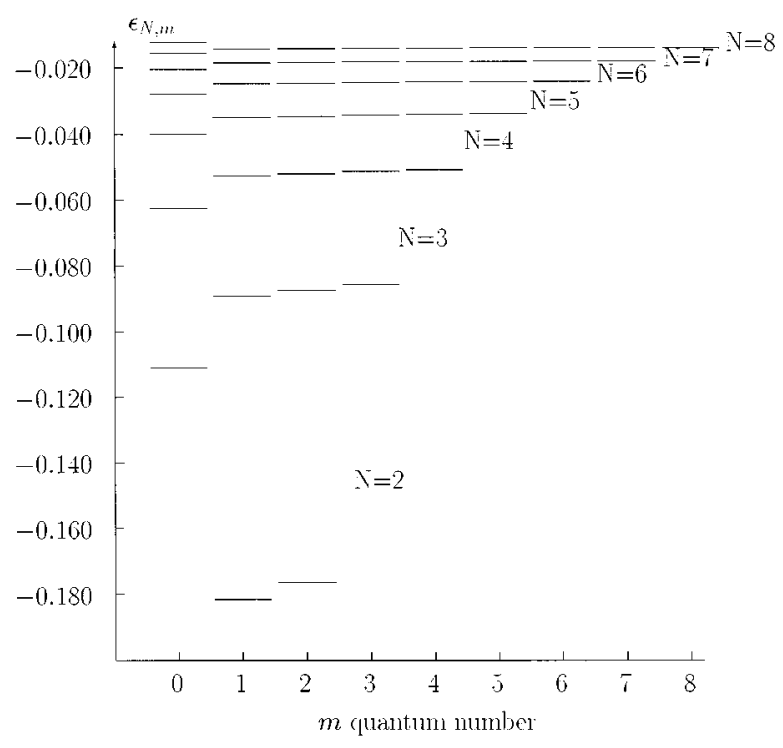

FIG. 2. The numerically calculated energy eigenvalues in the range $2 \leqslant N \leqslant 8$. The nondegeneracy in $m$ is clear.

$$
H_{m}^{\mathrm{adiab}}=-\frac{d^{2}}{d \rho^{2}}+\frac{m^{2}-1 / 4}{\rho^{2}}+\frac{2}{\rho} \frac{S_{z}}{\hbar},
$$

which for the bound component $\chi^{(-)}$is similar to the radial equation for a two-dimensional hydrogen atom (or a threedimensional one with a strange "angular momentum" of $\ell=m-1 / 2)$.

The eigenfunctions are then confluent hypergeometric functions, and the eigenvalues are

$$
\epsilon_{N, m}^{\mathrm{adiab}}=-\frac{1}{(N+1 / 2)^{2}}
$$

$N$ is analogous to the principal quantum number of hydrogen with the number of nodes in the radial wave function for a state with quantum numbers $(N, m)$ being $N-m$. We note that the system in the adiabatic limit, just like the hydrogen atom, can be solved by supersymmetric quantum mechanics $[10,12]$, which is reflected in the degeneracy in $m$ for a given value of $N$. We may factorize $H_{m}^{\text {adiab }}$ as explained in Eqs. (7)-(9), and $H_{m+1}^{\text {adiab }}$ is the supersymmetric partner of $H_{m}^{\text {adiab }}$. The ground state wave function may be found by solving the first-order differential equation

$$
A_{m} \chi^{(-)}=\left(\frac{d}{d \rho}-\frac{a_{m}}{\rho}+\frac{1}{a_{m}}\right) \chi^{(-)}=0,
$$

where we have introduced the notation

$$
a_{m}=m+1 / 2 \text {. }
$$

Except for normalization, the solution to (14) is

$$
\chi_{N=m, m}^{(-)}(\rho)=\rho^{a_{m}} \exp \left(-\frac{\rho}{a_{m}}\right) .
$$

Due to the supersymmetric connection between Hamiltonians of neighboring values of $m$, the excited states for angular momentum $m$ can be found by successive applications of $A^{\dagger} \mathrm{s}$ :

$$
\chi_{N, m}^{(-)}(\rho)=A_{m}^{\dagger} A_{m+1}^{\dagger} \cdots A_{N-1}^{\dagger} \chi_{N, N}^{(-)}(\rho) .
$$

The resulting wave function has the structure

$$
\chi_{N, m}^{(-)}(\rho)=\sum_{i=0}^{N-m} C_{N, m}^{i} \rho^{a_{m+i}} \exp \left(-\frac{\rho}{a_{N}}\right),
$$

where the coefficients $C_{N, m}^{i}$ are found by a recurrence relation

$$
C_{N, m}^{j}=\left\{\begin{array}{l}
1 \quad \text { for } j=0, \quad m=N \\
-\left(a_{m}+a_{m+j+1}\right) C_{N, m+1}^{j}+\frac{a_{m}+a_{N}}{a_{m} a_{N}} C_{N, m+1}^{j-1} \text { otherwise. }
\end{array}\right.
$$

In the limit where $m$ is large, the adiabatic approximation gives a good description of the system. In order to obtain results for any value of $(N, m)$, we study the system by numerical techniques.

\section{NUMERICAL RESULTS}

We have solved the problem numerically, and the resulting energy eigenvalues are not degenerate in $m$ for a given value of $N$, as they are in the spin-1/2 case.

Our numerical procedure is straightforward: the three coupled second-order radial differential equations are written as six coupled first-order differential equations and solved by a relaxation technique described in [16] with the energy eigenvalue introduced as a seventh variable. The program needs as input an initial guess for the three spinor components and their derivatives as well as the energy eigenvalue. Initially, we used input wave functions found from a numerical analysis similar to that described in the Appendix of [8], but our program turned out to work just as well with the wave function and energy eigenvalue obtained from the adiabatic limit.

The numerical eigenvalues were calculated for a number of different values for the computational parameters (e.g., number of integration mesh points and large $\rho$ integration limit, $\left.\rho_{\max }\right)$. From the variation in the resulting energies, we infer the accuracy of our numerical eigenvalues. We show in Fig. 1 the eigenfunction corresponding to quantum numbers $(N=8, m=7)$. The eigenfunctions are composed of all three components, and the contribution to the wave functions 
TABLE I. Numerically calculated energy eigenvalues $\epsilon_{N, m}$. The results for $m=0$ are analytically determined, other results are accurate to the last digit. We have varied $\rho_{\max }$ between $40(N+1 / 2)$ and $80(N+1 / 2)$, and the number of points between 5000 and 25000 in order to assess the accuracy.

\begin{tabular}{llllll}
\hline \hline$N$ & \multicolumn{1}{c}{$m=0$} & $m=1$ & $m=2$ & \multicolumn{1}{c}{$m=3$} & $m=4$ \\
\hline 4 & -0.0400 & -0.0527 & -0.052079 & -0.0512134 & -0.05076227 \\
3 & -0.0625 & -0.0891 & -0.087435 & -0.08555612 & \\
2 & -0.111111 & -0.1815 & -0.1762887 & & \\
1 & -0.2500 & -0.555 & & & \\
\hline \hline
\end{tabular}

from components that by themselves are unbound due to the sign on the "Coulomb" part of the potential, $2 S_{z} /(\hbar \rho)$, does not lead to unbound three-component spinors. The reason for this is the coupling between the spinor components through the action of the nondiagonal operator $S_{x}$.

In Fig. 2, we show the numerically calculated energy eigenvalues in the range $2 \leqslant N \leqslant 10$. For the record, some of these values are also listed in Table I, as well as energies for smaller values of $N$. As we go higher in $N$ the nondegeneracy in $m$ persists, as indicated by the results in Table II.

We have checked that the Hermiticity requirement, mentioned earlier and in Appendix A, is fulfilled for our numerically calculated spinor wave functions. This consistency check is especially important for the lowest energy states of $m=1$, since the wave function for this value of the total angular momentum has contributions from components with vanishing orbital momentum, for which the particle can pass "through" the wire and experience an abrupt change in the magnetic field.

\section{ANALYTIC APPROXIMATIONS}

In this section, we discuss our attempts to find an approximate analytic solution to the problem. First, let us digress on the symmetry properties of the system at hand.

\section{A. "Runge-Lenz" operator}

The possibility of solving the spin-1/2 system analytically by the use of techniques from supersymmetric quantum mechanics is related to the existence of an extra constant of motion analogous to the Runge-Lenz vector in the scalar hydrogen atom. This extra constant of motion is found in Ref. [14]. The corresponding operator has two components and in our notation it is given by

$$
\begin{aligned}
\alpha_{y}= & -\frac{1}{\rho}\left(\frac{S_{y}}{\hbar} \sin \phi-\frac{S_{z}}{\hbar} \cos \phi\right) \cos \phi \\
& +\frac{1}{2}\left[-i \frac{\partial}{\partial \rho_{z}} \frac{J_{x}}{\hbar}+\frac{J_{x}}{\hbar}\left(-i \frac{\partial}{\partial \rho_{z}}\right)\right], \\
\alpha_{z}= & \frac{1}{\rho}\left(\frac{S_{y}}{\hbar} \sin \phi-\frac{S_{z}}{\hbar} \cos \phi\right) \sin \phi \\
& +\frac{1}{2}\left[-i \frac{\partial}{\partial \rho_{y}} \frac{J_{x}}{\hbar}+\frac{J_{x}}{\hbar}\left(-i \frac{\partial}{\partial \rho_{y}}\right)\right] .
\end{aligned}
$$

Here $J_{x}$ is the total angular momentum along the wire, $J_{x}=S_{x}+L_{x}$, and $\rho_{z(y)}=\rho \sin \phi(\cos \phi)$. In the spin- $1 / 2$ case, the operators $\alpha_{y}, \alpha_{z}$, and $J_{x}$ are the generators for the dynamical symmetry group $\mathrm{O}(3)$.

Since the equations for both the spin- 1 and spin- $1 / 2$ systems have the same general structure, one might expect that if any extra constant of motion exists in the spin- 1 case, it would originate from operators with the same structure as $\alpha_{y}$ and $\alpha_{z}$ above. It turns out that the two operators in Eqs. (20) and (21) do not commute with the Hamiltonian for the spin-1 system. Also, they are not part of the generators for the Lie algebra of $\mathrm{O}(3)$. The reason is that the commutators taken between the operators, $H, \alpha_{x}, \alpha_{y}$, depend not only on commutators between the spin operators, but also on anticommutators like $\left\{S_{x}, S_{y}\right\}$ which vanish for spin-1/2, but not for any higher value of the spin. Therefore, it seems that the spin-1/2 system is unique in its symmetry properties, and so we cannot hope for an exact analytic solution of the spin-1 problem via supersymmetric quantum mechanics. Nevertheless, we have derived approximate analytic solutions based on the supersymmetry technique, as we shall now discuss.

\section{B. Perturbation on an approach with global supersymmetry}

Guided partly by the success of the supersymmetry technique for the spin-1/2 system and partly by the near-

TABLE II. Numerically calculated energy eigenvalues $\epsilon_{N, m}$ (except for $m=0$ ) for selected high lying energy eigenstates. We varied $\rho_{\max }$ between $90(N+1 / 2)$ and $180(N+1 / 2)$, and used $20000-25000$ points for each calculation.

\begin{tabular}{lllllll}
\hline \hline$N$ & \multicolumn{1}{c}{$m=0$} & \multicolumn{1}{c}{$m=3$} & \multicolumn{1}{c}{$m=6$} & \multicolumn{1}{c}{$m=15$} & $m=25$ & $m=40$ \\
\hline 25 & -0.001479 & -0.0015476 & -0.0015428 & -0.00153988 & -0.00153908 \\
30 & -0.0010406 & -0.0010806 & -0.0010779 & -0.00107614 & -0.00107568 \\
35 & $-7.7716 \times 10^{-4}$ & $-7.970 \times 10^{-4}$ & $-7.953 \times 10^{-4}$ & $-7.942 \times 10^{-4}$ & $-7.93933 \times 10^{-4}$ & $-6.098513 \times 10^{-4}$ \\
40 & $-5.9488 \times 10^{-4}$ & $-6.120 \times 10^{-4}$ & $-6.1086 \times 10^{-4}$ & $-6.1014 \times 10^{-4}$ & $-6.0995 \times 10^{-4}$ & - \\
\hline \hline
\end{tabular}


degeneracy observed for the numerical energy eigenvalues as indicated in Fig. 2, we try solving the spin-1 system by splitting the radial Hamiltonian $H_{m}$ into a Hamiltonian that is solvable by a supersymmetric factorization, and a perturbation. We write

$$
H_{m}=H_{m}^{\mathrm{SUSY}}+U_{m}
$$

and construct $H_{m}^{\mathrm{SUSY}}$ such that the supersymmetric partner of $H_{m}^{\mathrm{SUSY}}$ is $H_{m+1}^{\mathrm{SUSY}}$, i.e.,

$$
H_{m}^{\mathrm{SUSY}}=A_{m}^{\dagger} A_{m}+K_{m}, \quad H_{m}^{\mathrm{SUSY},(+)}=A_{m} A_{m}^{\dagger}+K_{m}=H_{m+1}^{\mathrm{SUSY}},
$$

where $A_{m}, A_{m}^{\dagger}$ are defined as in Eq. (8). With this construction there is a "global supersymmety" due to the direct connection between Hamiltonians for different angular momenta $m$. Moreover, we require the constant matrix $K_{m}$ to be diagonal in order to be able to identify an energy eigenvalue. Consider a normalizable solution $\chi_{N=m, m}(\rho)$ to the firstorder differential equation $A_{m} \chi_{m, m}(\rho)=0$. For such a spinor wave function, we have

$$
H_{m}^{\mathrm{SUSY}} \chi_{m, m}(\rho)=K_{m} \chi_{m, m}(\rho)=\epsilon_{m}^{\mathrm{SUSY}} \chi_{m, m}(\rho),
$$

provided $K_{m}$ is $\epsilon_{m}^{\mathrm{SUSY}}$ times the identity matrix. Consequently, $\chi_{m, m}(\rho)$ is an eigenfunction for $H_{m}^{\mathrm{SUSY}}$, and it is the ground state. Since $H_{m}^{\mathrm{SUSY}}$ and $H_{m+1}^{\mathrm{SUSY}}$ are supersymmetric partners, they have the same energy spectrum except for the ground state energy of $H_{m}^{\mathrm{SUSY}}$, and thus we know the entire energy spectrum of $H_{m}^{\mathrm{SUSY}}, H_{m+1}^{\mathrm{SUSY}}, H_{m+2}^{\mathrm{SUSY}}, \ldots$ from the factorization above.

First, note that the adiabatic approximation corresponds to such a supersymmetric factorization, but one in which $K_{m}$ is diagonal with two different energies, $\epsilon_{N, m}^{\text {adiab }}$ and 0 . There are three solutions to the ground state differential equation $A_{m} \chi=0$ which are in principle three-component wave functions, but since the superpotential $W_{m}(\rho)$ in $A_{m}$ is diagonal as well, each of the three solutions can be chosen to have just one nonvanishing component. Only one of the three solutions corresponds to a normalizable wave function. Thus we can relax our requirement on $K_{m}$ which needs not be proportional to the identity matrix. However, it must be diagonal, and two spinor components that are coupled by $W_{m}(\rho)$ must have similar diagonal elements in $K_{m}$. Our analysis indicates that the only useful factorization of the type discussed here (where $K_{m}$ is not proportional to the unity matrix) is the one corresponding to the adiabatic approximation.

To improve upon the adiabatic limit result, we may add a perturbation $U_{m}=H_{m}-H_{m}^{\text {adiab }}$. To first order, however, we will still find only one nonvanishing component in the spinor wave function since the components corresponding to the eigenvalues 0 and $+\hbar$ of $S_{z}$ are unbound in the adiabatic limit. The first-order contribution to the energy stems from a centrifugal barrier term in $U_{m}, S_{x}^{2} / \rho^{2}$, and we get for the first-order energy spectrum

$$
\epsilon_{N, m}^{\mathrm{adiab}}+\delta \epsilon_{N, m}=-\frac{1}{\left(N+\frac{1}{2}\right)^{2}}+\frac{1}{2 m\left(N+\frac{1}{2}\right)^{3}}, \quad m \neq 0,
$$

where the degeneracy in $m$ is lifted. We shall return to this result when comparing with the numerical energy eigenvalues.

For the more general supersymmetric Hamiltonians, we allow each component of $W_{m}(\rho)$ to have the structure $f_{1}(m) / \rho+f_{2}(m)$ in order to retrieve terms proportional to $1 / \rho$ and $1 / \rho^{2}$ in the supersymmetric Hamiltonian. The factors $f_{1}(m)$ and $f_{2}(m)$ are arbitrary real rational functions of $m$, of the form

$$
f_{i}(m)=\frac{\alpha_{i} m^{2}+\beta_{i} m+\delta_{i}}{\gamma_{i} m^{2}+\nu_{i} m+\eta_{i}}
$$

The parameters $\alpha_{i}, \beta_{i}$, etc., are arbitrary real constants; but to obtain the required supersymmetry [cf. Eq. (23)], the coefficients $\alpha_{i}$ and $\gamma_{i}$ must vanish. With this structure for $W_{m}(\rho)$, we find eight different supersymmetric models which have the required global supersymmetry and with $K_{m}$ proportional to the identity matrix. All of these models have three-component spinor eigenfunctions for $H_{m}^{\text {SUSY }}$.

Unfortunately, these supersymmetric models have one major flaw: when solving for the ground state wave function, $A_{m} \chi=0$, the unperturbed supersymmetric energy levels for a given set of the quantum numbers, $(N, m)$, turn out to be at least twofold degenerate. One may argue that this is due to the extra degree of freedom we added to the set of differential equations for the ground state wave function when requiring $K_{m}$ to have three degenerate eigenvalues instead of just the two encountered for the "true" $K_{m}$ [cf. Eq. (10)]. However, when we apply the perturbation $U_{m}=H_{m}-H_{m}^{\mathrm{SUSY}}$ to first order, one of the unperturbed degenerate eigenstates shifts slightly, whereas the other one (or two) become unbound and, consequently, it makes no sense to talk about a small perturbation. Remarkably, for most of these models and for a proper choice of the free parameters of the given model, the wave functions and first-order energies for the eigenstates that shift only slightly are in reasonable agreement with the numerical results. An example of such a model is described in Appendix B.

\section{Supersymmetric approach with local supersymmetry}

In another approach, in the spirit of Eq. (22), we look for a Hamiltonian $H_{m}^{\text {SUSY }}$ as before, but without the requirement that its supersymmetric partner be $H_{m+1}^{\mathrm{SUSY}}$. Such a local supersymmetric model exists, and we obtain nondegenerate eigenvalues for each state $(N, m)$, which to zeroth order are also not degenerate in $m$.

The details of this model are given in Appendix C, but the basic idea is to create a chain of supersymmetric Hamiltonians for each value of $m$ in the following fashion:

$$
\tilde{H}_{m}=\left(-\frac{d}{d \rho}+\tilde{W}_{m}(\rho)\right)\left(\frac{d}{d \rho}+\tilde{W}_{m}(\rho)\right)+\tilde{K}_{m}=\tilde{A}_{m}^{\dagger} \tilde{A}_{m}+\tilde{K}_{m},
$$

where $\tilde{H}_{m}$ is close to $H_{m}$ in some sense to be discussed in the Appendix, and 


$$
\begin{aligned}
& \tilde{H}_{m}^{(+)}=\tilde{A}_{m} \tilde{A}_{m}^{\dagger}+\tilde{K}_{m}=\tilde{A}_{m(1)}^{\dagger} \tilde{A}_{m(1)}+\tilde{K}_{m(1)}, \\
& \tilde{A}_{m(1)} \tilde{A}_{m(1)}^{\dagger}+\tilde{K}_{m(1)}=\tilde{A}_{m(2)}^{\dagger} \tilde{A}_{m(2)}+\tilde{K}_{m(2)},
\end{aligned}
$$

etc., where Eq. (28) defines the operators $\tilde{A}_{m(1)}, \tilde{A}_{m(2)}, \ldots$. Such a construction is possible when we work in the $m$-independent basis that corresponds to the large $m$ limit of the $m$-dependent basis of eigenstates that diagonalize $K_{m}$ [cf. Eq. (10)]. We find that in each case the superpotential of $\tilde{A}_{m(j)}, \tilde{W}_{m(j)}$, has the same structure as the original model superpotential $\tilde{W}_{m}$ [see Eqs. (27) and (C6)] and are thus still shape invariant potentials (cf. $[11,12]$ ).

The energy predictions from this model for the state with quantum numbers $(N, m)$ is

$$
\epsilon_{N, m}=-\left(\frac{a_{m}^{2}}{b_{m}\left(N+\frac{1}{2}\right)}\right)^{2},
$$

where

$$
b_{m}=m(m+1) .
$$

The expression (29) will be compared with the numerical results below. The wave functions corresponding to these energy eigenvalues have only one nonvanishing component, the $\chi^{(-)}$component, as is the case also in the adiabatic approximation. The ground state for angular momentum $m$ $(N=m)$ has the nonvanishing component

$$
\chi_{m, m}^{(-)}(\rho)=\rho^{a_{m}} \exp \left(-\frac{a_{m}}{b_{m}} \rho\right) .
$$

As explained earlier, we may find the excited states by successive application of $\tilde{A}^{\dagger}$ operators on corresponding ground state wave functions.

\section{COMPARISON}

Our analytic predictions will now be compared to the exact numerical results. In general, we expect the various approximate models of Sec. V to be in better agreement with the numerical results the larger the value of $m$, but for different reasons. The adiabatic approximation of Sec. III relies on the orbital period being large compared to the Larmor precession time. In a classical Coulomb potential, the orbital period increases as $r^{3 / 2}$ (Kepler's third law), whereas with a magnetic field varying as $1 / r$, the Larmor precession time increases proportional to $r$. Therefore, the larger the mean radius of the bound state of the atom around the wire, the better is the adiabatic approximation.

For a given set of states with principal quantum number $N$, the mean radius varies roughly as $\langle\rho\rangle \sim(N+1 / 2)^{2}$, since the wave functions are approximately $(N+1 / 2)$-order polynomials times $\exp [-\rho /(N+1 / 2)]$. Consequently, we expect the adiabatic approximation to be good for all states in the set with sufficiently large principal quantum number $N$, e.g., for $N \geqslant 10$.

On the other hand, the model with local supersymmetry, as described in Sec. V C and in Appendix C, is an approximation to the original Hamiltonian in Eq. (5) for large values

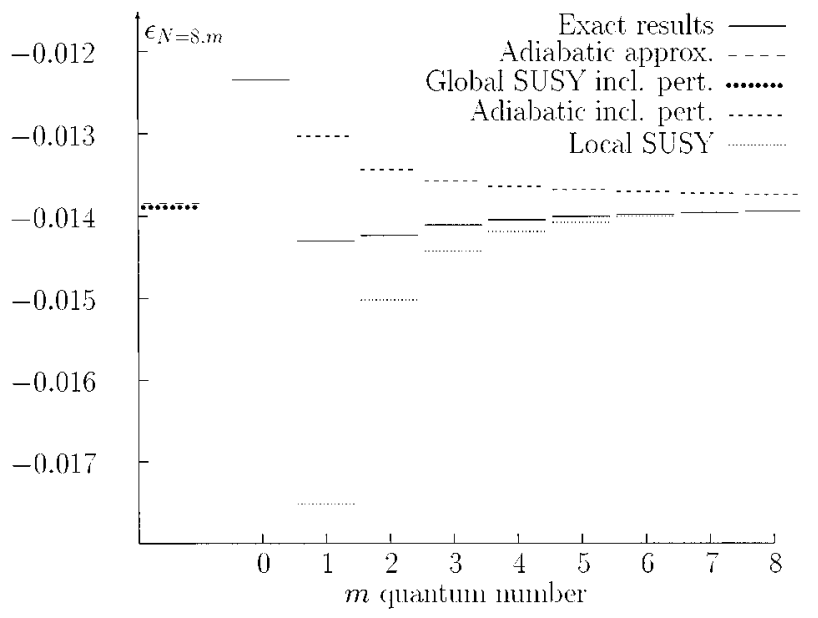

FIG. 3. Energy eigenvalues for $N=8$. The solid lines give the numerical values except for $m=0$, for which the exact analytic result is given. The dashed line to the left of the figure is the result of the adiabatic approximation without perturbation, and the shortdashed lines give the results of the adiabatic approximation including the first-order perturbation. The line with circles to the left represents the global supersymmetric model including the firstorder perturbation as described in Appendix B, and the dotted lines give the model with local supersymmetry as described in Appendix C.

of $m$. Thus, we expect a good agreement with the numerical results only for large $m$ 's, e.g., $m \geqslant 10$.

In Fig. 3, we show the energy eigenvalues for a set $N=8$. The numerical results are shown as solid lines and the adiabatic approximation of Eq. (13) is shown as a longdashed line to the left. Note that it predicts the same energy eigenvalue for all of the states shown. This is also the case for the line with circles (not valid for $m=0$ ) showing the result of the perturbative calculation to first order on the supersymmetric model with global supersymmetry described in Appendix B, Eq. (B9). The adiabatic approximation plus perturbation to first order, Eq. (25), is shown as short-dashed lines. Finally, the predictions from our third type of supersymmetric model, with local supersymmetry, $\epsilon_{N, m}$, in Eq. (29), are shown as dotted lines. We note that the predictions of the adiabatic approximation get worse when the perturbation is added, and that the predictions of the model of Sec. V C are very good for the larger values of $m$. In Fig. 4, we have increased $N$ to 15 , to show that the analytic models get better with increasing $m$ or $N$. Once again, the model with local supersymmetry gives very good predictions for the states with largest $m$.

To compare wave functions, we show in Fig. 5 the three components of the wave function for $N=8, m=7$. The wave function of the adiabatic approximation, and that of the model with local supersymmetry, have only one nonvanishing component, whereas the wave function corresponding to the bound energy eigenvalue in Eq. (B9) has all three components. Note, though, that the $\chi^{(+)}$component of the spinor has an extra node. The different predictions for $\chi^{(-)}$are almost indistinguishable on the figure.

In order to better see agreement between the analytic models and the numerical results for increasing quantum numbers, we show in Fig. 6 the wave functions for 


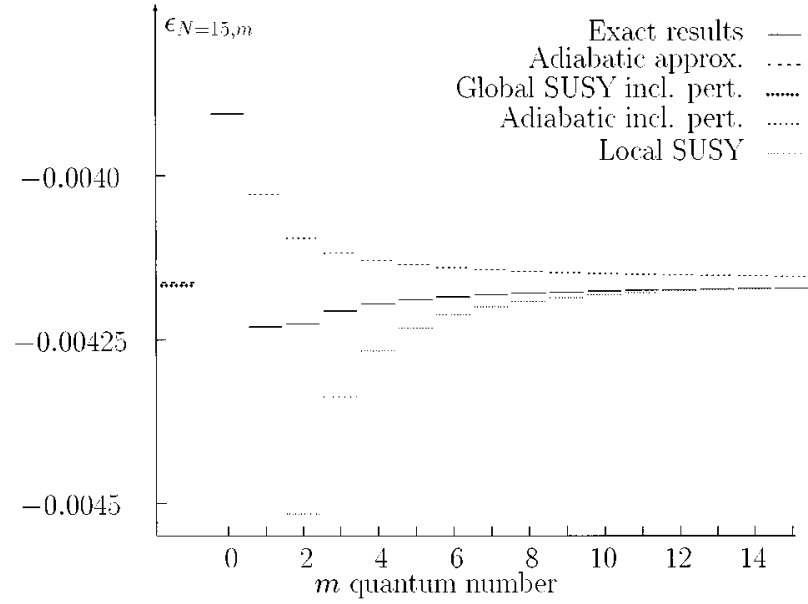

FIG. 4. The same as Fig. 3, but for $N=15$. We have omitted the prediction for $m=1$ for the model with local supersymmetry $\left(\epsilon_{N, m=1} \simeq-0.0053\right)$ in order to be able to distinguish the different energy predictions for the higher values of $m$.

$N=30, m=25$. Note that the adiabatic approximation is almost perfect for the $\chi^{(-)}$component of the spinor, and all the different predictions are almost indistinguishable. In general, as $m$ increases, the relative sizes of the different spinor components change such that the $\chi^{(-)}$component dominates, followed by the $\chi^{(0)}$ component.

\section{LIFETIMES}

The bound states of the particle around the wire can decay in two ways. First, the magnetic moment couples to the electromagnetic field of the vacuum, and we have a decay

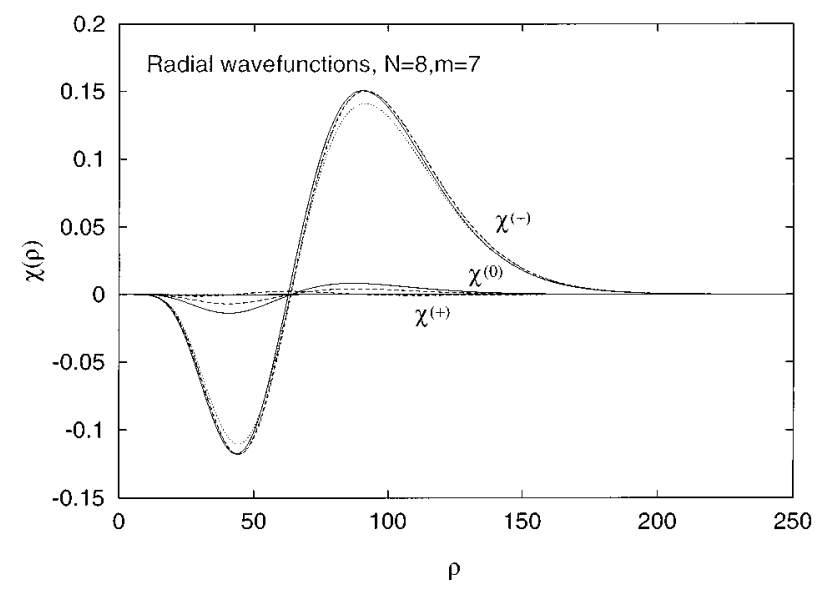

FIG. 5. The components of the radial wave function $\chi(\rho)$ for $N=8, m=7$, as calculated by the different models discussed. The numerical results are shown as solid lines (the same as Fig. 1). The long-dashed lines represent the model with global supersymmetry. We show the zeroth-order wave function that is still bound when the first-order perturbation is included. The wave function corresponds to the model described in Appendix B. For this model, the $\chi^{(+)}$ component is comparable in size to the $\chi^{(0)}$ component, and it has an extra node. The adiabatic approximation is shown as a shortdashed line. In this case only the $\chi^{(-)}$component is nonvanishing. The dotted line shows the $\chi^{(-)}$component of the model with local supersymmetry, as described in Appendix C.

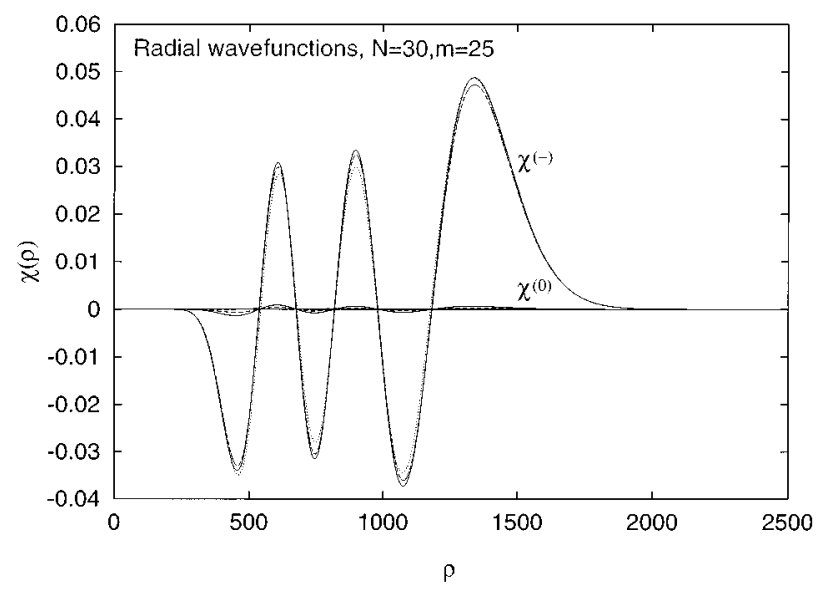

FIG. 6. As Fig. 5, but for $N=30, m=25$. The $\chi^{(+)}$component is not visible on this scale and the different predictions for $\chi^{(-)}$are almost indistinguishable.

mechanism quite similar to the electric dipole decay of atomic states. In addition, due to the finite thickness of the wire in the experiments, an atom in a bound state can hit the wire and get absorbed.

We can estimate the time $\tau_{N, m}^{a b s}$ before an atom in a bound state of quantum numbers $(N, m)$ hits the wire,

$$
\tau_{N, m}^{a b s}=\frac{2 \pi / \omega_{N, m}}{P_{N, m}}
$$

where $\omega_{N, m}$ is the frequency associated with the transverse energy eigenvalue, and $\hbar \omega_{N, m}=\epsilon_{N, m} C^{2} /\left(2 r_{0}\right)$ [cf. Eq. (6)]. $P_{N, m}$ is the probability that the atoms occupy the region $0 \leqslant \rho \leqslant R_{\text {wire }} / r_{0}$, where $R_{\text {wire }}$ is the radius of the wire, i.e.,

$$
P_{N, m}=\int_{0}^{R_{\text {wire }} / r_{0}}\left|\chi_{N, m}(\rho)\right|^{2} d \rho .
$$

For the state $(N=40, m=25)$, and for a current of $I=400 \mu \mathrm{A}$ through a wire of radius $0.25 \mu \mathrm{m}$, the binding energy is $E \simeq-9 \times 10^{-9} \mathrm{eV}, P_{N, m} \simeq 6 \times 10^{-5}$ and the lifetime is $\tau_{40,25}^{a b s} \simeq 72 \mathrm{msec}$. The time to absorption increases rapidly as one goes to larger $m$ values within the same manifold $N$, and it is very large for "Rydberg" states like $(N=40, m=40)$ where $P_{N, m} \simeq 8 \times 10^{-32}$. For states with a considerable overlap with the wire our analysis needs to be modified. For these states we need to take into account that the magnetic field dependence on $r$ is different inside the wire. As in the numerical calculations in [8], we assume that the current density in the wire is constant, and consequently the magnetic field inside the wire is proportional to $r$. This change is readily included in our numerical routine, and for a state at approximately the same energy eigenvalue as a given state $(N, m)$ in the infinitely thin wire case, we observe that the effect of the finite thickness is to move the probability distribution further out and thus to decrease the probability for the atom to hit the wire. Also, a finite wire thickness causes a decrease in the number of nodes for the bound states, as illustrated in Fig. 7. Note that in calculating the bound states we did not consider any effects of the wire surface. 


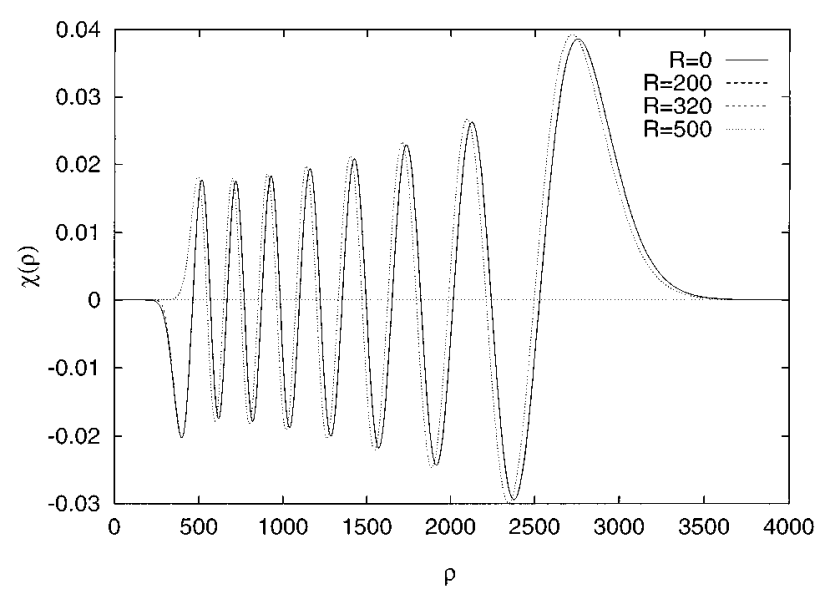

FIG. 7. The dominating component, $\chi^{(-)}(\rho)$, of the radial wave function corresponding to $N=40, m=25$. The solid line shows the result for an infinitely thin wire, the long-dashed line is the result for a wire of thickness $R=R_{\text {wire }} / r_{0}=200$, the shortdashed line corresponds to $R=320$, and the dotted line to $R=500$. The energy eigenvalues are $\epsilon_{N, m}=-6.0995 \times 10^{-4}$; $-6.0991 \times 10^{-4} ;-6.0972 \times 10^{-4}$; and $-6.1642 \times 10^{-4}$, respectively.

The lifetime due to the interaction with the vacuum magnetic field can be calculated with the dipole approximation applied for the two bound degrees of freedom perpendicular to the wire. This calculation is described in Appendix D, where one main result is a selection rule in $m$, Eq. (D7), $\Delta m=0, \pm 1$. This selection rule implies that, e.g., a state $(m, m)$ can decay only to $(m, m-1)$ and $(m-1, m-1)$.

As for the electric dipole transitions in atoms, the transition probability for a given transition scales with the frequency of the transition cubed, cf. Eq. (D19). With the parameters we are considering, these frequencies are very small. Furthermore, compared to electric dipole transitions in atoms, the expression for the magnetic dipole transition probability contains an extra factor of $\alpha^{2}$, where $\alpha$ is the fine structure constant. Consequently, the transition probabilities are very small, and we obtain lifetimes for states like $(N=40, m=40)$ of $\tau_{40,40}^{v a c} \simeq 6 \times 10^{30} \mathrm{~s}$ for a current of $I=400 \mu \mathrm{A}$ through a wire of radius $R_{\text {wire }}=0.25 \mu \mathrm{m}$. This number is large for all other states we have looked at, e.g., $\tau_{40,25}^{v a c} \simeq 1 \times 10^{29} \mathrm{~s}$ and $\tau_{8,8}^{v a c} \simeq 3 \times 10^{24} \mathrm{~s}$.

A more serious concern is the effect of blackbody radiation from the hot wire on the cold atoms. The wires envisioned in the experiment have small radii and with the required current the wire heats up considerably. Let us define $p_{\mathbf{k}} d^{3} \mathbf{k}$ as the radiated energy flux out through a unit area of the blackbody for a radiation mode in the volume element of size $d^{3} \mathbf{k}$ around $\mathbf{k}$; cf., e.g., [18]. We want to calculate the intensity of radiation, $I_{\mathbf{k}}$, associated with modes around $\mathbf{k}$ hitting an atom at a distance $r$ from the wire. If we view the wire as an infinitely long slab of width $2 \pi R_{\text {wire }}$, the contribution from an area $\mathscr{b}$ on the wire, centered around a point $x_{0}$, is given as $p_{\mathbf{k}} d^{3} \mathbf{k} \cos ^{2} \theta, A l r^{2}$. Here $\theta$ denotes the angle between the surface normal of the slab and the direction from $x_{0}$ to the atom. To get $I_{\mathbf{k}}$ we integrate over all points $x_{0}$ on an infinitely long wire, thus integrating over all values of the angle $\theta$. We take the conservative viewpoint that any photon absorption will take the atom out of the trapped ground state
Zeeman sublevel. If we consider only one possible transition, of resonant frequency $\omega_{0}$ corresponding to the wavelength $\lambda$, and with linewidth $\Gamma$ (which in radial frequency equals $2 \pi / \tau$, where $\tau$ is the lifetime of the upper state of the transition) the rate of absorption, $W_{\mathbf{k}}$, of a photon in mode $\mathbf{k}$ is

$$
W_{\mathbf{k}}=\frac{\Gamma}{2} \frac{I_{\mathbf{k}} / I_{\text {sat }}}{1+4\left(\frac{\omega_{0}-|\mathbf{k}| c}{\Gamma}\right)^{2}+I_{\mathbf{k}} / I_{\text {sat }}} \simeq \frac{\Gamma}{2} \frac{I_{\mathbf{k}} / I_{\text {sat }}}{1+4\left(\frac{\omega_{0}-|\mathbf{k}| c}{\Gamma}\right)^{2}} .
$$

We have assumed that the intensity $I_{\mathbf{k}}$ is small compared to the transition saturation intensity $I_{\text {sat }}=(\pi h c) /\left(3 \lambda^{3} \tau\right)$. For the total absorption probability we then find

$$
W_{\mathrm{abs}}=\frac{24 \lambda^{3} \pi}{c^{3} \tau^{4}} \frac{R_{\mathrm{wire}}}{r} \int_{0}^{\infty} \frac{x^{3} d x}{\left[1+4\left(\frac{\omega_{0}}{\Gamma}-x\right)^{2}\right]\left[\exp \left(\frac{\hbar \Gamma}{k_{B} T} x\right)-1\right]}
$$

The integral is calculated numerically for a given temperature of the wire (which depends on the current density in the wire).

The transition considered here is the electric dipole transition in $\mathrm{Na}$ used for laser cooling, $3 S_{1 / 2}(F=2) \rightarrow 3 P_{3 / 2}(F=3)$ with $\lambda=589 \mathrm{~nm}$ and $\tau=16 \mathrm{~ns}$. The magnetic dipole transitions of the bound states around the wire are very weak, and the effect of the blackbody radiation due to these transitions is negligible.

The shortest absorption time, $t_{\mathrm{abs}}=1 / W_{\mathrm{abs}}$, appears at $r=R_{\text {wire }}$. For a wire radius of $R_{\text {wire }}=0.5 \mu \mathrm{m}$, and a current of $I=400 \mu \mathrm{A}$ in a copper wire, the wire temperature is around $400 \mathrm{~K}$ and we find the absorption time to be $t_{\mathrm{abs}} \simeq 1.3 \times 10^{4} \mathrm{~s}$ or $3.6 \mathrm{~h}$. If the wire radius is decreased by a factor of 2 , the temperature increases to around $850 \mathrm{~K}$, and the absorption time is $t_{\mathrm{abs}} \simeq 231 \mathrm{~s}$ or almost $4 \mathrm{~min}$. We are also considering using polished rhenium wire, and the temperature corresponding to optimum current and radius is on the order of 1000-1200 K. In this case the tail of the blackbody radiation spectrum reaches out to the optical resonance, and consequently, the absorption time is much shorter, ranging from $t_{\mathrm{abs}} \simeq 5 \mathrm{~s}$ at $1000 \mathrm{~K}$ to $t_{\mathrm{abs}} \simeq 0.09 \mathrm{~s}$ at $1200 \mathrm{~K}$. These numbers are based upon an assumption that the wire has an emissivity of 1 ; with more realistic values on the order of 0.2 for polished rhenium, the absorption time increases by a factor of 5. Although these absorption times are rather small, rhenium wire is convenient because it can also be used as a sensitive hot wire detector for the alkali-metal atoms.

From this analysis, it seems clear that the magnetic dipole transitions do not limit the experiments. The absorption on the wire may impose a limit, but there will be atoms bound in states of high enough angular quantum number $m$ that the absorption is negligible and so we do not think absorption is a problem either. The blackbody radiation is clearly a limiting factor for the experiments using a high temperature wire, whereas for lower temperatures the limiting factor for the lifetime of the bound atoms around the wire is probably collisions with the background gas. For sodium trapped in a magnetic quadrupole trap with a background gas pressure of $3.75 \times 10^{-11}$ Torr, the lifetime due to background gas collisions is observed to be $30 \mathrm{~s}$ [19]. 


\section{CONCLUSION}

We have shown that for the system composed of a spin-1 magnetic particle coupled to a current carrying wire, bound states of two-dimensional motion around the wire exist, and the system is thus a candidate waveguide for matter waves. Previously, this waveguide was studied for a spin- $1 / 2$ particle with an exact analytic solution [5], but it turns out that the symmetry properties of the system change with the spin, and as a result we have not been able to find an exact analytic solution for the spin-1 system. A numerical solution of the problem shows that the energy eigenvalues are not degenerate in the angular quantum number $m$, but are almost degenerate.

We have presented approximate models with analytic solutions in our attempts to describe the spin- 1 system. The near-degeneracy of the numerical energy eigenvalues and the success of the supersymmetric factorization for the spin-1/2 system mean that it is useful to find a model Hamiltonian which can be solved exactly by supersymmetric factorization, and treat the difference between the exact Hamiltonian and the model Hamiltonian as a perturbation. It remains puzzling, however, why this method of finding a "nearby" Hamiltonian with global supersymmetry does not lead to any convincing results. The most disturbing outcome of these calculations is that it seems that in order to find an analytic spinor wave function with three nonvanishing components, we have to face a series of degenerate unperturbed eigenlevels. The first-order perturbation imposes an energy splitting for some of the levels which is inconsistant with perturbation theory. However, we find the states, for which the first-order energy corrections are small, useful in comparison with our other results.

In a slightly different approach we find a nearby Hamiltonian with local supersymmetry. The local supersymmetry implies that the degeneracy in $m$ is lifted already to zeroth order in the difference between the true Hamiltonian and the model Hamiltonian. In our comparison of the different supersymmetric models with the exact results, we note that for large values of $m$, the local supersymmetric model gives very good predictions for the energy eigenvalues. This is certainly true for the ground state and the first few excited states for a given value of $m$. Unfortunately, the model gives only one nonvanishing component of the spinor wave function.

Our studies of the different models leave an unanswered but very interesting question: what is the small physical parameter responsible for the small breaking of the symmetry we observe in the numerical energy eigenvalues?

The waveguide we have discussed in this paper is feasible to study experimentally as well. We expect to observe bound states around the wire, in the quantum regime, with laser cooled atoms. Sodium in the ground state has total angular momentum $F=1$ and thus corresponds to the calculations presented here. In the bound state with quantum numbers $(N=40, m=40)$ it has a binding energy of $E_{t}=9 \times 10^{-9} \mathrm{eV}$ for a wire of radius $R_{\text {wire }}=0.25 \mu \mathrm{m}$ and with a current of $I=400 \mu \mathrm{A}$. Accordingly, it should be possible to bind laser cooled sodium atoms of a kinetic temperature around 25 $\mu \mathrm{K}$ in such a state. These low temperatures are readily accessible via polarization gradient laser cooling. In "Ryd- berg" bound states $(N=m)$, corresponding to large angular momenta, the lifetime is limited by collisions of the bound atoms with the background gas. For lower lying states and for hot wires, we expect the decay of the bound states to be dominated by blackbody radiation emitted from the hot wire.

\section{ACKNOWLEDGMENTS}

We thank the Rowland Institute for Science for its continuing support. K.B.-S. also acknowledges enlightening discussions with A. Lesniewski and thanks the Carlsberg Foundation and the Danish Natural Science Research Council for financial support.

\section{APPENDIX A: HERMITICITY}

In order to address the question of Hermiticity of the Hamiltonian, particularly of the kinetic energy operator as $\rho \rightarrow 0$, we perform an analysis similar to that presented by Pauli [13]. The main differences are that in our case, the wave functions are $(2 s+1)$-component spinors (threecomponent for spin 1), and the bound motion is in two dimensions rather than three. Hermiticity implies

$$
\int v^{\dagger} \mathscr{H} u d^{3} r=\int(\mathscr{H} v)^{\dagger} u d^{3} r
$$

for any set $u$ and $v$ of spinor eigenfunctions of the general Hamiltonian $\mathscr{H}$. To prove the validity of Eq. (A1), we first consider the equality

$$
\int_{V}\left[v^{\dagger} \mathscr{H} b u-(\mathscr{H} v)^{\dagger} u\right] d^{3} r=\int_{\sigma} \mathbf{j}\left(v^{\dagger}, u\right) \cdot d \mathbf{a},
$$

where the surface integral over $\mathbf{j}$ gives the (vanishing) probability flux. The probability current density $\mathbf{j}$ is proportional to

$$
\mathbf{j}\left(v^{\dagger}, u\right) \propto \sum_{i=1}^{2 s+1}\left(v_{i}^{*} \nabla u_{i}-u_{i} \nabla v_{i}^{*}\right)
$$

where the spinor components of $v$ and $u$ are denoted by $v_{1}, v_{2}, \ldots, u_{1}, u_{2}, \ldots$ To study the Hermiticity of the Hamiltonian, we will apply Eq. (A2) with $\sigma$ surrounding the singular point of the potential. The Hermiticity of the Hamiltonian in the basis of eigenstates is ensured if the surface integral of the current density vanishes when the area of $\sigma$ vanishes.

For our two-dimensional problem, we consider a small circle of radius $r$ around the singularity $r=0$. The corresponding path integral is

$$
I_{\ell}=r \sum_{i=1}^{2 s+1} \int_{0}^{2 \pi}\left[v_{i}^{*} \frac{\partial u_{i}}{\partial r}-u_{i} \frac{\partial v_{i}^{*}}{\partial r}\right] d \phi .
$$

For the spinor components we use $u(v)_{i}=e^{i m \phi} \chi_{i}^{u(v)}(r) /$ $\sqrt{r}$, and get

$$
I_{\ell}=\delta_{m_{u}, m_{v}} \sum_{i=1}^{2 s+1}\left[\chi_{i}^{v} \frac{d \chi_{i}^{u}}{d r}-\chi_{i}^{u} \frac{d \chi_{i}^{v}}{d r}\right],
$$


where we have chosen the radial spinor components $\chi_{i}(r)$ to be real. For $m_{u}=m_{v}$, we need to consider the radial wave functions. Since we only consider normalizable wave functions, $\int_{0}^{\infty} \Sigma_{i} \chi_{i}^{2} d r$ is finite, each spinor component $\chi_{i}(r)$ must be of the form $\chi_{i}(r)=r^{\alpha}\left[f_{i}(r)+g_{i}(r) \ln (r)\right]$ for $r \rightarrow 0$ $(\alpha>-1 / 2)$, where $f_{i}$ and $g_{i}$ are functions of $r$ that are regular at the origin. We find for the terms in square brackets in Eq. (A4)

$$
\chi_{i}^{v} \frac{d \chi_{i}^{u}}{d r}-\chi_{i}^{u} \frac{d \chi_{i}^{v}}{d r}=r^{2 \alpha-1}\left[f_{i}^{u} g_{i}^{v}-f_{i}^{v} g_{i}^{u}+r \mathscr{F}_{i}(r)\right]
$$

where $\mathscr{F}_{i}(r)$ is a combination of derivatives of $f_{i}$ 's and $g_{i}$ 's and $r \mathscr{F}_{i}(r)$ vanishes at the origin. Consequently, when $2 \alpha-1$ is positive, the Hermiticity requirement is fulfilled, and we thus require the power $\alpha$ to be strictly larger than $1 / 2$. However, if there are no logarithmic contributions to the spinor components the Hermiticity requirement is fulfilled for any power $\alpha>0$.
We checked that our numerically calculated spinor components do indeed vanish with a power of $r$ larger than $1 / 2$.

\section{APPENDIX B: EXAMPLE OF PERTURBATION TREATMENT FOR THE APPROACH WITH GLOBAL SUPERSYMMETRY}

In this appendix, we present one example of the supersymmetric models with a perturbation as described in Sec. $\mathrm{V}$ B. We work in the basis of eigenfunctions $|0\rangle,| \pm\rangle$ for the operator $S_{z}$ with eigenvalues $0, \pm \hbar$,

$$
\mathbf{e}_{1}=|-\rangle, \quad \mathbf{e}_{2}=|0\rangle, \quad \mathbf{e}_{3}=|+\rangle
$$

We have chosen the free parameters in $H_{m}^{\text {SUSY }}$ such that the perturbation $U_{m}$ has as few elements as possible, used as a naive criterion for $U_{m}$ being "small." In this case, the equations look like an "expanded" spin-1/2 system. The superpotential reads

$$
W_{m}(\rho)=\left\{\begin{array}{ccc}
-\frac{\mathscr{b}_{m}}{\rho}+\frac{\beta_{0}}{\mathscr{b}_{m}} & \frac{1}{\sqrt{2} \rho} & 0 \\
\frac{1}{\sqrt{2} \rho} & -\frac{\mathscr{b}_{m}}{\rho}-\frac{\beta_{0}}{\mathscr{b}_{m}} & \frac{1}{\sqrt{2} \rho} \\
0 & \frac{1}{\sqrt{2} \rho} & -\frac{\mathscr{b}_{m}}{\rho}+\frac{\left|\beta_{0}\right|}{\mathscr{b}_{m}}
\end{array}\right\},
$$

$\mathscr{C}_{m}=m+\alpha$, and $\epsilon_{m}^{\mathrm{SUSY}}=-\beta_{0}^{2} /(m+\alpha)^{2} . \alpha$ and $\beta_{0}$ are arbitrary real constants. We choose $\beta_{0}>0$ and $\alpha=1 / 2$, finding the perturbation to be

$$
U_{m}=\left\{\begin{array}{ccc}
\frac{2\left(-1+\beta_{0}\right)}{\rho} & 0 & 0 \\
0 & -\frac{2 \beta_{0}}{\rho} & 0 \\
0 & 0 & \frac{2\left(1+\beta_{0}\right)}{\rho}
\end{array}\right\} .
$$

The perturbation introduces no divergences stronger than Coulombic terms and it is independent of the value of $m$, $U_{m} \equiv U$. To have the smallest number of terms in $U$, we choose $\beta_{0}=1$. Note then that $\epsilon_{m=N}^{\mathrm{SUSY}}$ equals $\epsilon_{N, m}^{\mathrm{adiab}}$ in (13).

The ground-state wave functions have the form

$$
\begin{gathered}
\chi_{m, m}^{(1)}(\rho)=K_{1} \rho^{a_{m-1}} \exp \left(-\frac{\rho}{a_{m}}\right)\left(\begin{array}{c}
1+\frac{2 \rho}{a_{m}} \\
\sqrt{2} \\
2 \rho \\
1+\frac{2 \rho}{a_{m}}
\end{array}\right), \\
\chi_{m, m}^{(2)}(\rho)=K_{2} \rho^{a_{m}} \exp \left(-\frac{\rho}{a_{m}}\right)\left(\begin{array}{c}
1 \\
0 \\
-1
\end{array}\right),
\end{gathered}
$$

where $K_{1}$ and $K_{2}$ are normalization constants. Also for the excited states, as we shall see below, a state $\chi_{N, m}^{(i)}(\rho)$ has the form of a polynomial in $\rho$ with powers ranging from $(m-1 / 2)$ to $(N+1 / 2)$ times an exponentially decreasing factor. There are no logarithmic terms in the wave functions, and thus even with the requirements of Hermiticity of the 
kinetic energy operator, we can accept any positive power of $\rho$ in the wave function. Consequently, the model is good for any $m \geqslant 1$.

Due to the supersymmetric connection between $H_{m}^{\text {SUSY }}$ and $H_{m+1}^{\text {SUSY }}$, we can easily find the excited states via Eq. (17). The supersymmetry implies that the $n$th excited state, $\chi_{N, m}^{(i)}(\rho)$, for $H_{m}^{\mathrm{SUSY}}$ has the same energy as the ground state, $\chi_{N, N}^{(i)}(\rho)$, for $H_{N}^{\text {SUSY }}$. By applying $A^{\dagger}$ s to $\chi^{(2)}$, we find

$$
\chi_{N, m}^{(2)}(\rho)=K^{N, m} \sum_{j=0}^{N-m} \mathscr{C}_{j}^{N, m} \rho^{a_{m+j}} \exp \left(-\frac{\rho}{a_{N}}\right)\left(\begin{array}{c}
1 \\
0 \\
-1
\end{array}\right),
$$

where the coefficients $\mathscr{C}_{j}^{N, m}$ are found from the same recurrence relation (19) as the "adiabatic" one. Similarly, we find for $\chi^{(1)}$

$$
\chi_{N, m}^{(1)}(\rho)=\kappa^{N, m} \sum_{j=0}^{N-m+1} \mathbf{C}_{j}^{N, m} \rho^{a_{m-1+j}} \exp \left(-\frac{\rho}{a_{N}}\right),
$$

where the coefficient vectors $\mathbf{C}_{j}^{N, m}$ are found from the recurrence relation

$$
\mathbf{C}_{j}^{N, m}=\left\{\begin{array}{l}
\mathbf{v}_{1}+\sqrt{2} \mathbf{v}_{2} \quad \text { for } j=0, m=N \\
\frac{2}{a_{m}} \mathbf{v}_{1} \quad \text { for } j=0, m=N-1 \\
-\left(a_{m}+a_{m+j}\right) \mathbf{C}_{j}^{N, m+1}+\frac{1}{a_{N}} \mathbf{C}_{j-1}^{N, m+1}+\frac{1}{\sqrt{2}}\left[\left(\mathbf{C}_{j}^{N, m+1} \cdot \mathbf{v}_{1}\right) \mathbf{v}_{2}+\left(\mathbf{C}_{j}^{N, m+1} \cdot \mathbf{v}_{2}\right) \mathbf{v}_{1}\right] \\
\quad+\frac{1}{a_{m}}\left[\left(\mathbf{C}_{j-1}^{N, m+1} \cdot \mathbf{v}_{1}\right) \mathbf{v}_{1}-\left(\mathbf{C}_{j-1}^{N, m+1} \cdot \mathbf{v}_{2}\right) \mathbf{v}_{2}\right] \quad \text { otherwise, }
\end{array}\right.
$$

with the vectors $\mathbf{v}_{1}=(1,0,1)$ and $\mathbf{v}_{2}=(0,1,0)$.

We find matrix elements of $U$ in Eq. (B3) in the basis of eigenfunctions $\chi_{N, m}^{(1)}$ and $\chi_{N, m}^{(2)}$ of each twofold degenerate level of energy eigenvalue $\epsilon_{N}^{\text {SUSY }}$, and diagonalize the perturbation matrix. By calculating a few examples $(N \leqslant 8)$, we find that the supersymmetric energy plus first-order perturbation yields

$$
\epsilon_{N}^{\mathrm{SUSY}}+\delta \epsilon_{N, m}=\frac{1}{(N+1 / 2)^{2}}-\frac{2}{(N+1 / 2) \sqrt{N(N+1)}}
$$

corresponding to the zeroth-order wave function

$$
\chi_{N, m}(\rho)=\frac{1}{\sqrt{2}}\left[\chi_{N, m}^{(1)}(\rho)+\chi_{N, m}^{(2)}(\rho)\right]
$$

We also find a series of nonbound states, of energy

$$
\epsilon_{N}^{\mathrm{SUSY}}+\delta \boldsymbol{\epsilon}_{N, m}^{\prime}=\frac{1}{(N+1 / 2)^{2}}+\frac{2}{(N+1 / 2) \sqrt{N(N+1)}} .
$$

Note that for this supersymmetric model, to first order in the perturbation, the energy degeneracy in $m$ persists. This is not the case for other models we have studied. In most other models the energies that are still bound after the first-order perturbation show the same trend with $m$ for a given value of $N$ as the energies predicted from the adiabatic approximation with the first-order perturbation included.

\section{APPENDIX C: MODEL WITH LOCAL SUPERSYMMETRY}

Here we give the details of the supersymmetric model discussed in Sec. V C. In order to describe this model, we return to the supersymmetric factorization of the original Hamiltonian, Eq. (5). We look at the $m$-dependent basis of eigenstates that diagonalize $K_{m}$, and go to the limit of large $m$ in order to find an $m$-independent set of basis states. In terms of eigenstates of $S_{z} / \hbar$, this basis is

$$
\mathbf{e}_{1}=|0\rangle, \quad \mathbf{e}_{2}=\frac{1}{\sqrt{2}}(|-\rangle+|+\rangle), \quad \mathbf{e}_{3}=\frac{1}{\sqrt{2}}(|-\rangle-|+\rangle)
$$

In this basis, $K_{m}$ and $W_{m}(\rho)$ are

$$
\begin{gathered}
W_{m}(\rho)=\left\{\begin{array}{ccc}
-\frac{a_{m}}{\rho} & \frac{1}{\rho} & \frac{1}{2 b_{m}} \\
\frac{1}{\rho} & -\frac{a_{m}}{\rho} & \frac{a_{m}}{b_{m}} \\
K_{m}=\left\{\begin{array}{ccc}
\frac{1}{2 b_{m}} & \frac{a_{m}}{b_{m}} & -\frac{a_{m}}{\rho}
\end{array}\right\}, \\
-\frac{a_{m}}{2 b_{m}^{2}} & -\frac{a_{m}}{2 b_{m}^{2}} & 0 \\
0 & -\frac{a_{m}^{2}}{b_{m}^{2}} & 0 \\
0 & 0 & \varepsilon_{1}
\end{array}\right\},
\end{gathered}
$$


where only one of the diagonal elements in $K_{m}$ equals an eigenvalue; cf. Eq. (10). In these equations, $b_{m}=m(m+1)$; cf. Eq. (30). It turns out that if we approximate $K_{m}$ by $\tilde{K}_{m}$ equal to $\varepsilon^{\prime}(m)$ times the unit matrix, with

$$
\varepsilon^{\prime}(m)=-\left(\frac{a_{m}}{b_{m}}\right)^{2}
$$

and the superpotential $W_{m}(\rho)$ by

$$
\begin{aligned}
& \tilde{W}_{m}(\rho)=\left\{\begin{array}{ccc}
-\frac{a_{m}}{\rho} & 0 & 0 \\
0 & -\frac{a_{m}}{\rho} & \frac{a_{m}}{b_{m}} \\
0 & \frac{a_{m}}{b_{m}} & -\frac{a_{m}}{\rho}
\end{array}\right\} \\
& =\frac{a_{m}}{\rho} \mathbf{1}+\left\{\begin{array}{ccc}
0 & 0 & 0 \\
0 & 0 & c_{m} \\
0 & c_{m} & 0
\end{array}\right\} \text {, }
\end{aligned}
$$

it is possible to create a chain of supersymmetric Hamiltonians in the following fashion:

$$
\begin{gathered}
\tilde{H}_{m}=\left(-\frac{d}{d \rho}+\tilde{W}_{m}(\rho)\right)\left(\frac{d}{d \rho}+\tilde{W}_{m}(\rho)\right)+\tilde{K}_{m}=\tilde{A}_{m}^{\dagger} \tilde{A}_{m}+\tilde{K}_{m}, \\
\tilde{H}_{m}^{+}=\tilde{A}_{m} \tilde{A}_{m}^{\dagger}+\tilde{K}_{m}=\tilde{A}_{m(1)}^{\dagger} \tilde{A}_{m(1)}+\tilde{K}_{m(1)}, \\
\tilde{A}_{m(1)} \tilde{A}_{m(1)}^{\dagger}+\tilde{K}_{m(1)}=\tilde{A}_{m(2)}^{\dagger} \tilde{A}_{m(2)}+\tilde{K}_{m(2)},
\end{gathered}
$$

etc. In each case, the superpotential has the structure of $\tilde{W}_{m}$ in Eq. (C6) (shape invariance), such that the superpotential $\tilde{W}_{m(j)}$ in $\tilde{A}_{m(j)}$ equals $\tilde{W}_{m}$ with the replacements

$$
a_{m} \rightarrow a_{m+j}, \quad c_{m} \rightarrow \frac{a_{m}}{a_{m+j}} c_{m} .
$$

Likewise, we get for $\tilde{K}_{m(j)}$

$$
\tilde{K}_{m(j)}=\left\{\begin{array}{ccc}
\varepsilon^{\prime}(m) & 0 & 0 \\
0 & \varepsilon^{\prime}(m)+\delta e_{m, j} & 0 \\
0 & 0 & \varepsilon^{\prime}(m)+\delta e_{m, j}
\end{array}\right\},
$$

with

$$
\delta e_{m, j}=\frac{j a_{m}^{2}\left(a_{m}+a_{m+j}\right)}{b_{m}^{2} a_{m+j}^{2}} .
$$

The energy eigenvalue for a given state of quantum numbers $(N, m)$ is then $\epsilon_{N, m}=\varepsilon^{\prime}(m)+\delta e_{m, N-m}$; cf. Eq. (29). The wave functions corresponding to these energy eigenvalues must have vanishing first components in the basis (C1), and when we transform back to the basis of eigenstates of $S_{z} / \hbar$, only the $|-\rangle$ component is nonvanishing, as in the adiabatic approximation. This nonvanishing component for the ground state is given in Eq. (31), and the excited states may be found by application of $A^{\dagger}$ operators on the groundstate wave function.

\section{APPENDIX D: DECAY DUE TO INTERACTION WITH THE VACUUM FIELD}

We want to calculate the decay of a bound eigenstate of the Hamiltonian in Eq. (1) (with the magnetic field from the wire, $\mathbf{B}=\mathbf{B}_{\text {wire }}$ ), due to the perturbation

$$
H_{\text {int }}=-\boldsymbol{\mu} \cdot \mathbf{B}_{v a c} .
$$

The calculation resembles that of the transition probability for electric dipole transitions in atoms found in many textbooks, e.g., [20], but has some special features due to the cylindrical symmetry of the problem, and we have chosen to give the full derivation in this appendix.

With the vacuum field quantized in a cubic box of volume $V=L^{3}$, the magnetic field of the vacuum, $\mathbf{B}_{v a c}$, is

$$
\begin{aligned}
\mathbf{B}_{v a c}(\mathbf{R})= & i \sum_{\mathbf{k}, \lambda=1,2} \sqrt{\frac{2 \pi \hbar \omega_{\mathbf{k}}}{V}}\left(\hat{\mathbf{k}} \times \boldsymbol{\varepsilon}_{\mathbf{k} \lambda}\right)\left(a_{\mathbf{k} \lambda} e^{i \mathbf{k} \cdot \mathbf{R}}\right. \\
& \left.-a_{\mathbf{k} \lambda}^{\dagger} e^{-i \mathbf{k} \cdot \mathbf{R}}\right),
\end{aligned}
$$

where the sum is over all allowed values for $\mathbf{k}$ in the volume $V$, and $\lambda$ indicates the sum over the two polarization vectors $\boldsymbol{\varepsilon}_{\mathbf{k} \lambda}$ perpendicular to $\mathbf{k}$. The frequency is $\omega_{\mathbf{k}}=|\mathbf{k}| c$, and $a_{\mathbf{k} \lambda}$ and $a_{\mathbf{k} \lambda}^{\dagger}$ are the usual annihilation and creation operators for the particular mode. The vector $\hat{\mathbf{k}}$ is a unit vector in the direction of $\mathbf{k}$.

In order to calculate the transition probability from a state of quantum numbers $(N, m)$ and longitudinal wave number $\kappa$, with zero photons in the vacuum field, to a state $\left(N^{\prime}, m^{\prime}\right)$ and $\kappa^{\prime}$ with one photon in any mode in the vacuum field, we need to consider the matrix element

$$
\mathscr{C}=-i g \mu_{B} \sum_{\mathbf{k}, \lambda=1,2} \sqrt{\frac{2 \pi \hbar \omega_{\mathbf{k}}}{V}}\left|\psi_{N^{\prime}, m^{\prime}, \kappa^{\prime}}, n_{\mathbf{k} \lambda}=1\right|\left(\hat{\mathbf{k}} \times \boldsymbol{\varepsilon}_{\mathbf{k} \lambda}\right) \cdot \frac{\mathbf{S}}{\hbar} a_{\mathbf{k} \lambda}^{\dagger} e^{-i \mathbf{k} \cdot \mathbf{R}}\left|\psi_{N, m, \kappa}, 0\right\rangle,
$$

where we have inserted the expression for the magnetic moment, Eq. (2), and neglected the annihilating part of the magnetic field, since it does not contribute. This matrix element consists of a transverse part and a longitudinal part, where we refer to the coordinates perpendicular to or in the direction of the wire. For the transverse part, it is valid to make a dipole approximation in the magnetic field: even though the radii of the bound states are macroscopic (on the order of micrometers), the binding energies, and thus the wave numbers corresponding to on-shell transitions, are very small $\left(10^{-9} \mathrm{eV}\right)$, and $\mathbf{k}_{t} \cdot \mathbf{r}$ is much smaller than 1. However, we cannot make a dipole approximation for the longitudinal part along the wire. Instead, the matrix element $\mathscr{C}$ is found by replacing $\exp (-i \mathbf{k} \cdot \mathbf{r})$ in Eq. (D3) by $\exp \left(-i k_{x} x\right)$. We may also readily perform the integral over the field degrees of freedom. 
To evaluate the matrix element further, consider first the transverse part of it; insert expression (4) for $\psi_{N, m, \kappa}=\exp \left(-i S_{x} \phi / \hbar\right) \Psi_{N, m, \kappa}$, and observe the transverse matrix element, $\mathscr{C l}_{t}$,

$$
\begin{aligned}
\mathscr{M}_{t} & =\left(\hat{\mathbf{k}} \times \boldsymbol{\varepsilon}_{\mathbf{k} \lambda}\right) \cdot \frac{1}{2 \pi}\left\langle e^{i m^{\prime} \phi} \frac{\chi_{N^{\prime}, m^{\prime}}(r)}{\sqrt{r}}\left|e^{i S_{x} \phi / \hbar} \frac{\mathbf{S}}{\hbar} e^{-i S_{x} \phi / \hbar}\right| e^{i m \phi} \frac{\chi_{N, m}(r)}{\sqrt{r}}\right\rangle \\
& =\left(\hat{\mathbf{k}} \times \boldsymbol{\varepsilon}_{\mathbf{k} \lambda}\right) \cdot \mathscr{P} .
\end{aligned}
$$

The factor of $1 /(2 \pi)$ in Eq. (D4) is put explicitly for normalization of the $\phi$-dependent part of the wave function. In (D5), we have introduced the shorthand notation $\mathscr{S}$ for the relevant spin-vector matrix element, with components $\mathscr{S}_{x}$, $\mathscr{S}_{y}$, and $\mathscr{S}_{z}$. Now, note the effect of the rotation with $\exp \left(-i S_{x} \phi / \hbar\right)$ :

$$
\begin{gathered}
e^{i S_{x} \phi / \hbar} \frac{S_{x}}{\hbar} e^{-i S_{x} \phi / \hbar}=\frac{S_{x}}{\hbar}, \\
e^{i S_{x} \phi / \hbar} \frac{S_{y}}{\hbar} e^{-i S_{x} \phi / \hbar}=\cos \phi \frac{S_{y}}{\hbar}-\sin \phi \frac{S_{z}}{\hbar}, \\
e^{i S_{x} \phi / \hbar} \frac{S_{z}}{\hbar} e^{-i S_{x} \phi / \hbar}=\cos \phi \frac{S_{z}}{\hbar}+\sin \phi \frac{S_{y}}{\hbar},
\end{gathered}
$$

implying that in terms of $\phi$, the operator in the matrix element varies as 1 or $e^{ \pm i \phi}$. Consequently, there is a selection rule in the quantum number $m$ :

$$
\Delta m=0, \pm 1 \text {. }
$$

Thus, for a ground state at a given $m$, there are only two possible decays: $(m, m) \rightarrow(m, m-1)$ leading to an almost vanishing photon energy, and $(m, m) \rightarrow(m-1, m-1)$. [In the spin-1/2 case there is only one decay channel for a state $(m, m)$, namely, to $(m-1, m-1)$, since $(m, m)$ and $(m, m-1)$ are degenerate.]

From Fermi's golden rule the probability $W$ for the particular transition $(N, m) \rightarrow\left(N^{\prime}, m^{\prime}\right)$ is

$$
\begin{aligned}
W= & \sum_{\lambda} \int_{\mathbf{k}} \int_{\kappa^{\prime}} \frac{2 \pi}{\hbar}|\mathscr{C l}|^{2} f_{v}(\mathbf{k}) d \mathbf{k} f_{x}\left(\kappa^{\prime}\right) d \kappa^{\prime} \\
& \times \delta\left(E_{N^{\prime}, m^{\prime}, \kappa^{\prime}}+\hbar \omega_{\mathbf{k}}-E_{N, m, \kappa}\right),
\end{aligned}
$$

where $f_{v}$ and $f_{x}$ are the densities of states for the vacuum field and the free motion along the wire. We are only interested in the bound-state-to-bound-state part of the transition. The energy of a state $(N, m, \kappa), E_{N, m, \kappa}$, is

$$
E_{N, m, \kappa}=\epsilon_{N, m} \frac{C^{2}}{2 r_{0}}+\frac{\hbar^{2} \kappa^{2}}{2 M}
$$

For the energy difference we introduce a wave number, $\tilde{k}\left(\kappa^{\prime}\right)$,

$$
\begin{aligned}
\tilde{k}\left(\kappa^{\prime}\right)= & \frac{E_{N, m, \kappa}-E_{N^{\prime}, m^{\prime}, \kappa^{\prime}}}{\hbar c}=\left(\epsilon_{N, m}-\epsilon_{N^{\prime}, m^{\prime}}\right) \frac{C^{2}}{2 \hbar c r_{0}} \\
& +\frac{\hbar\left[\kappa^{2}-\left(\kappa^{\prime}\right)^{2}\right]}{2 M c} .
\end{aligned}
$$

The density of states for the vacuum field is

$$
f_{v}(\mathbf{k}) d \mathbf{k}=\frac{V}{(2 \pi)^{3}} k_{t} d k_{x} d k_{t} d \theta
$$

Here we have explicitly separated the wave vector into a longitudinal part, $k_{x}$, and a transverse part, signified by the coordinates $k_{t}$ and $\theta$. For the longitudinal motion, if we quantize in a one-dimensional box of length $L$ the number of modes within the interval $[\kappa, \kappa+d \kappa]$ is $f_{x}(\kappa) d \kappa=(L / 2 \pi) d \kappa$.

We may now calculate the longitudinal part of the matrix element. The integral is straightforward: we have to integrate $(1 / L) \exp \left[i\left(\kappa-\kappa^{\prime}-k_{x}\right) x\right]$ from 0 to $L$. In order to get the transition probability, we square the matrix element and, as we in principle will look at the limit $L \rightarrow \infty$, write

$$
\lim _{L \rightarrow \infty}\left|\mathscr{C l}_{x}\right|^{2}=\lim _{L \rightarrow \infty} \frac{\sin ^{2}[L(\Delta \kappa / 2)]}{2 \pi L(\Delta \kappa / 2)^{2}} \frac{2 \pi}{L}=\lim _{L \rightarrow \infty} \frac{2 \pi}{L} \delta(\Delta \kappa) .
$$

Here $\Delta \kappa$ is shorthand for $\kappa-\kappa^{\prime}-k_{x}$.

We now insert the previous results in expression (D8) to get

$$
W=\int_{-\infty}^{\infty} d k_{x} \int_{0}^{\infty} k_{t} d k_{t} \int_{0}^{2 \pi} d \theta \sum_{\lambda} \frac{g^{2} \mu_{B}^{2}}{2 \pi} \omega_{\mathbf{k}}\left|\left(\hat{\mathbf{k}} \times \boldsymbol{\varepsilon}_{\mathbf{k} \lambda}\right) \cdot \mathscr{P}\right|^{2} \frac{1}{\hbar c} \delta\left(\tilde{k}\left(\kappa-k_{x}\right)-|\mathbf{k}|\right),
$$

where we have already used the $\delta$ function in Eq. (D12) to integrate over $\kappa^{\prime}$, and have changed the $\delta$ function in energy to a $\delta$ function in $|\mathbf{k}|$. 
Consider now the sum over polarizations, $\lambda$. The photon wave vector and the two perpendicular polarization vectors can be represented in Cartesian coordinates as

$$
\begin{aligned}
\mathbf{k} & =\mathbf{e}_{x} k_{x}+\mathbf{e}_{y} k_{t} \cos \theta+\mathbf{e}_{z} k_{t} \sin \theta, \\
\boldsymbol{\varepsilon}_{\mathbf{k} 1} & =\frac{1}{|\mathbf{k}|}\left(\mathbf{e}_{x} k_{t}-\mathbf{e}_{y} k_{x} \cos \theta-\mathbf{e}_{z} k_{x} \sin \theta\right), \\
\boldsymbol{\varepsilon}_{\mathbf{k} 2} & =\mathbf{e}_{y} \sin \theta-\mathbf{e}_{z} \cos \theta,
\end{aligned}
$$

and since

$$
\sum_{\lambda}\left|\left(\hat{\mathbf{k}} \times \varepsilon_{\mathbf{k} \lambda}\right) \cdot \mathscr{S}\right|^{2}=\left|\varepsilon_{\mathbf{k} 1} \cdot \mathscr{P}\right|^{2}+\left|\varepsilon_{\mathbf{k} 2} \cdot \mathscr{S}\right|^{2}
$$

we may now integrate over $\theta$, and obtain

$$
\int_{0}^{2 \pi} d \theta\left(\left|\boldsymbol{\varepsilon}_{\mathbf{k} 1} \cdot \mathscr{S}\right|^{2}+\left|\boldsymbol{\varepsilon}_{\mathbf{k} 2} \cdot \mathscr{S}\right|^{2}\right)=2 \pi\left(\frac{k_{t}^{2}\left|\mathscr{S}_{x}\right|^{2}+k_{x}^{2}\left(\left|\mathscr{S}_{y}\right|^{2}+\left|\mathscr{S}_{z}\right|^{2}\right) / 2}{k_{t}^{2}+k_{x}^{2}}+\frac{\left|\mathscr{S}_{y}\right|^{2}+\left|\mathscr{S}_{z}\right|^{2}}{2}\right) .
$$

Finally, let us elaborate on the remaining $\delta$ function in Eq. (D13), which ensures the energy conservation. Denote $f\left(k_{x}\right)=\tilde{k}\left(\kappa-k_{x}\right)-|\mathbf{k}|$, and use the property of the $\delta$ function, $\delta(f(x))=\Sigma_{i}\left|1 / f^{\prime}\left(x_{i}\right)\right| \delta\left(x-x_{i}\right)$, where $f\left(x_{i}\right)=0$. Also note that $\tilde{k}\left(\kappa-k_{x}\right)=k_{\epsilon}+k_{x}\left[\hbar\left(2 \kappa-k_{x}\right) /(2 M c)\right]$, where $k_{\epsilon}$ is shorthand for $\left(\epsilon_{N, m}-\epsilon_{N^{\prime}, m^{\prime}}\right) C^{2} /\left(2 \hbar c r_{0}\right)$. Here, $\hbar \kappa / M$ is the velocity of the bound atom in its direction of free motion, and $\hbar k_{x} / M$ is a velocity of similar size, much smaller than the velocity of light $c$. The "transverse" velocity $\hbar k_{t} / M$ is even smaller. It is therefore a good approximation to perform the following calculations to first order in $\hbar \kappa /(M c)$, $\hbar k_{t} /(M c)$, and $\hbar k_{x} /(M c)$ only. To first order in these three small numbers, we find two nodes for the function $f\left(k_{x}\right)$, $k_{x}^{ \pm}$,

$$
k_{x}^{ \pm} \simeq k_{\epsilon} \frac{\hbar \kappa}{M c} \pm \sqrt{k_{\epsilon}^{2}-k_{t}^{2}}\left(1-\frac{\hbar k_{\epsilon}}{2 M c}\right), \quad k_{t}^{2} \leqslant k_{\epsilon}^{2},
$$

and, again to lowest order in $\hbar /(M c)$ times any wave number, $\kappa, k_{\epsilon}$, or $k_{t}$, one finds for $\left|1 / f^{\prime}\left(k_{x}^{ \pm}\right)\right|$,

$$
\left|\frac{1}{f^{\prime}\left(k_{x}^{ \pm}\right)}\right| \simeq \frac{k_{\epsilon}}{\sqrt{k_{\epsilon}^{2}-k_{t}^{2}}} \pm \frac{\hbar \kappa}{M c}-\frac{\hbar}{2 M c} \frac{2 k_{\epsilon}^{2}-k_{t}^{2}}{\sqrt{k_{\epsilon}^{2}-k_{t}^{2}}}, k_{t}^{2} \leqslant k_{\epsilon}^{2} .
$$

Inserting these last results in the $\delta$ function, and carrying out the integral over $k_{x}$, we are then finally left with an integral over $k_{t}$ between 0 and $k_{\epsilon}$, or an integral over $u=k_{t} / k_{\epsilon}$ from 0 to 1 . We find

$$
\begin{aligned}
W \simeq & \frac{g^{2} \mu_{B}^{2}}{\hbar} k_{\epsilon}^{3}\left[\frac{2}{3}\left|\mathscr{S}_{x}\right|^{2}\left(2-\frac{\hbar k_{\epsilon}}{M c}\right)\right. \\
& \left.+\frac{4}{3}\left(\left|\mathscr{S}_{y}\right|^{2}+\left|\mathscr{S}_{z}\right|^{2}\right)\left(1-\frac{\hbar k_{\epsilon}}{M c}\right)\right] .
\end{aligned}
$$

Note that the lifetime of a given state $(N, m, \kappa)$, to this order in $\hbar /(M c)$ times any wave number involved, is independent of $\kappa$. The transition probability varies with the frequency $\omega=k_{\epsilon} c$ as $\omega^{3}$, as in the electric dipole transition between atomic states.
[1] See, e.g., the special issue of J. Opt. Soc. Am. B 6 (11) (1989), edited by S. Chu and C. Wieman.

[2] Proceedings of the Enrico Fermi Summer School, Course CXVIII, 1991, edited by E. Arimondo, W. D. Phillips, and F. Strumia (North-Holland, Amsterdam, 1992).

[3] P. Lett, R. Watts, C. Westbrook, W. Phillips, P. Gould, and H. Metcalf, Phys. Rev. Lett. 61, 169 (1988).

[4] C. Salomon, J. Dalibard, W. D. Phillips, A. Clairon, and S. Guellati, Europhys. Lett. 12, 683 (1990).

[5] L. V. Hau, J. A. Golovchenko, and M. M. Burns, Phys. Rev. Lett. 74, 3138 (1995).
[6] L. V. Hau, M. M. Burns, and J. A. Golovchenko, Phys. Rev. A 45, 6468 (1992).

[7] J. Schmiedmayer, Appl. Phys. B 60, 169 (1995).

[8] R. Blümel and K. Dietrich, Phys. Rev. A 43, 22 (1991).

[9] E. Witten, Nucl. Phys. B 188, 513 (1981).

[10] R. Dutt, A. Khare, and U. P. Sukhatme, Am. J. Phys. 56, 163 (1988).

[11] L. Gendenshtein, Pis'ma Zh. Éksp. Teor. Fiz. 38, 299 (1983) [JETP Lett. 38, 356 (1983)].

[12] F. Cooper, A. Khare, and U. Sukhatme, Phys. Rep. 251, 267 (1995). 
[13] W. Pauli, in General Principles of Quantum Mechanics (Springer-Verlag, Berlin, 1980), pp. 44,45.

[14] G. P. Pron'ko and Yu. G. Stroganov, Zh. Éksp. Teor. Fiz. 72, 2048 (1977) [Sov. Phys. JETP 45, 1075 (1977)].

[15] J. Schmiedmayer, Phys. Rev. A 52, R13 (1995).

[16] W. H. Press, B. P. Flannery, S. A. Teukolsky, and W. T. Vetterling, Numerical Recipes (Cambridge University Press, Cambridge, 1994).
[17] A. I. Voronin, Phys. Rev. A 43, 29 (1991).

[18] F. Reif, Fundamentals of Statistical and Thermal Physics (McGraw-Hill, New York, 1965), pp. 373-378.

[19] K. B. Davis, M.-O. Mewes, M. A. Joffe, M. R. Andrews, and W. Ketterle, Phys. Rev. Lett. 74, 5202 (1995).

[20] M. Weissbluth, Atoms and Molecules (Academic Press, San Diego, 1978), Chap. 23. 